\title{
MULTI-LAYER EMBEDMENT OF CONDUCTIVE AND NON-CONDUCTIVE PDMS FOR ALL-ELASTOMER MEMS
}

\author{
Jonathan M. Engel, Nannan Chen, Kee Ryu, Saunvit Pandya, Craig Tucker, Yingchen Yang, Chang Liu \\ Micro Actuators, Sensors, and Systems Group, University of Illinois at Urbana-Champaign \\ Urbana, IL USA
}

\begin{abstract}
PDMS (polydimethylsiloxane) elastomer is widely used in MEMS. However, PDMS is non-conductive and as a result is used in mostly structural applications. We report methods for monolithic integration of conductive and non-conductive PDMS for realizing wholly polymer-based devices with embedded elastomer wires, electrodes, heaters, and sensors. In this work we demonstrate elastomer strain gauges, capacitive pressure sensors, as well as microfluidic channels with integrated heaters and sensors. The process uses a series of PDMS patterning, micromolding, and bonding techniques with conductive PDMS features made by mixing with multiwall carbon nanotubes (MWNT).
\end{abstract}

\section{INTRODUCTION}

Traditionally, elastomers such as PDMS have played a large but mainly structural role in MEMS, serving as protective layers, encapsulants, valve diaphragms, fluidic channel structures, and so forth. However, a number of "active" devices have been made using modified elastomers, including organic vapor sensors [1], liquid sensors [2], force sensitive resistors [3], and ultrasonic emitters [4]. These devices use elastomers mixed with solid fillers, such as carbon black, MWNT, or metallic powders to give the resulting composite material the desired properties. Researchers have also captured metal films in PDMS layers to create elastomer tactile sensors [5]. Most recently, work has been done to capture in-situ grown MWNT in PDMS to create strain gauges and field emission devices [6].

The goal of our work is to create devices that can be handled directly, take advantage of the unique characteristics of composite elastomers, and enable applications that require conformal and robust materials. To develop a general new class of processes and structures to meet this goal, we take advantage of a process for precision patterning of thin film elastomers [7]. By combining patterning technique spin casting and molding, we have realized a number of all-elastomer devices with embedded conductors and sensors such as shown in Figure 1.

\section{DESIGN AND FABRICATION}

In order to realize functional regions of conductive PDMS in concert with structural insulating PDMS, we have developed a fabrication process as shown in Figure 2. The process begins with the vapor coating of chlorotrimethylsiloxane (CTMS) on the substrate to assist in the release of the final elastomer assembly (Figure 2a). Next, photoresist (PR) is spun and patterned to define the molds for the conductive PDMS (Figure 2b). PDMS is then mixed with multiwalled carbon nanotubes (MWNT) in order to make a conductive composite. The ratio of MWNT to PDMS elastomer is chosen depending on the desired application and performance of the device. In the case of simple conductors for capacitive sensors or resistive heaters, a large amount of MWNT may be added to increase the conductivity of the composite. In the case of strain or force sensitive devices, a lower loading of MWNT is desired to increase sensitivity. Details of the conductivity of PDMS and MWNT composites can be found in [8]. For the devices presented here, $10 \%$ by weight MWNT is mixed with Sylgard-184 PDMS.

Once the MWNT and PDMS have been mixed, the composite is applied to the PR mold and patterned as detailed in [7]. The patterned elastomer is cured at $90^{\circ} \mathrm{C}$ for 10 minutes, and the $\mathrm{PR}$ mold removed in acetone (Figure 2d). The conductive PDMS structures are then captured by either spin or pour casting unmodified PDMS around them as shown in Figure 2e. The resulting assembly is cured at $90^{\circ} \mathrm{C}$ for 30 minutes in a leveled oven before being peeled from the substrate (Figure 2f).

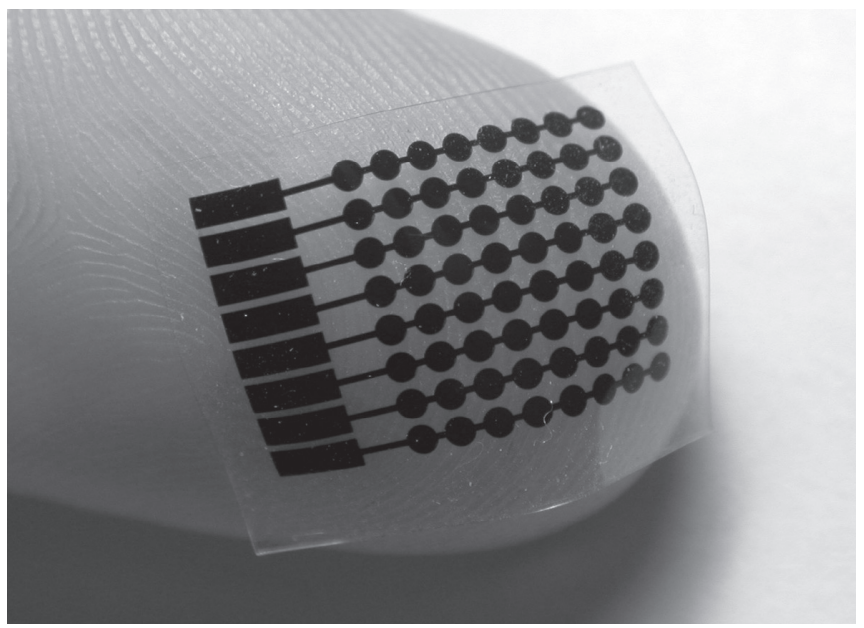

(a)

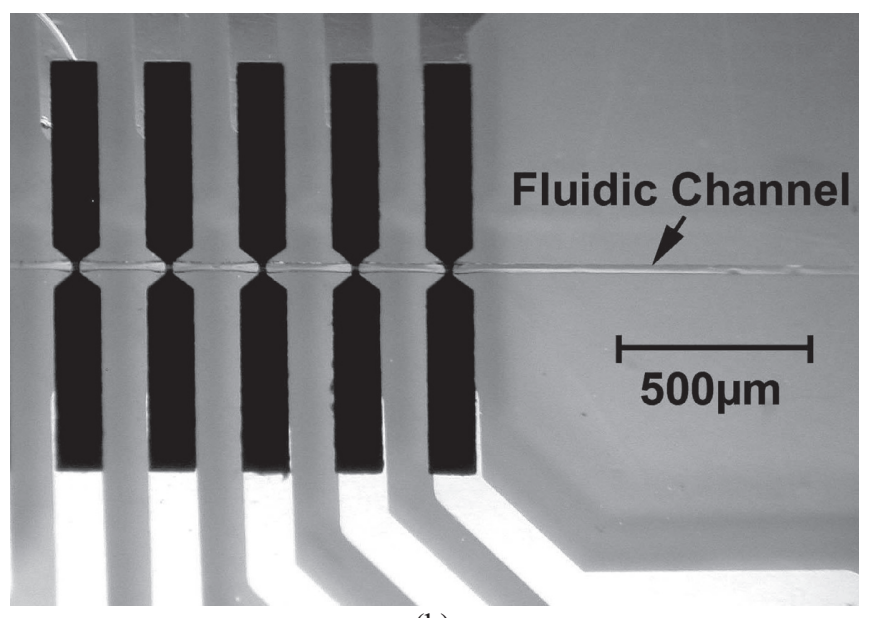

(b)

Figure 1: a) Photo of PDMS tactile sensor sheet. Black parts are made of conductive PDMS and embedded in transparent, nonconductive PDMS skin. b) Micrograph of PDMS microfluidic channel with embedded conductive elastomer devices that cross the channel (running horizontally). 


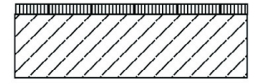

(a)

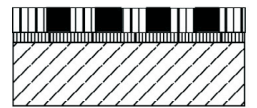

(c)

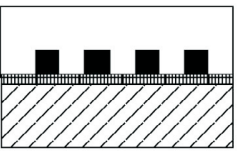

(e)

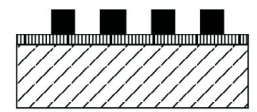

(d)

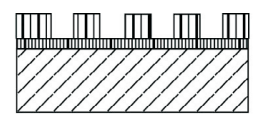

(b)

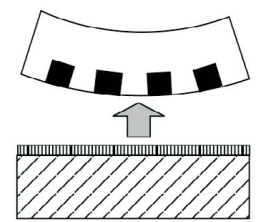

(f)
Substrate

CTMS
Figure 2: Schematic diagram of a single-layer general fabrication process: a) vapor coating substrate with CTMS (chlorotrimethylsiloxane), a chemical agent to facilitate later release in step $f$, b) patterning photoresist (PR) mold, c) applying $M W N T$ loaded PDMS and removing excess using a blade, d) removing $P R$ mold, leaving precision patterned functional PDMS behind, e) spin casting unmodified PDMS, f) and finally peeling the PDMS with embedded sensors.

This process can be combined with other traditional elastomer patterning techniques such as used for defining micro-fluidic or pneumatic channels (Figure 1b). Multiple layers can also be combined to create complex devices such as capacitive pressure sensors. We have applied this approach to produce a number of new devices - elastomer strain gauges, channels with embedded flow-rate sensors, and a soft capacitive tactile sensor are demonstrated.

\section{RESULTS AND DISCUSSION}

In simplest form, a strip of conductive elastomer is embedded as a strain gauge in unmodified PDMS (Figure 3a). Applied strain alters the average spacing between conductive particles and therefore the resistance reading (Figure $3 b$ ). In contrast to existing semiconductor and metal strain gauges, our all-elastomer strain gauge can repeatably measure large strains $(>1 \%)$. Embedded in the insulating elastomer, the gauge undergoes the same strain as the bulk PDMS, surviving large deformations typical of elastomers. Figure $3 \mathrm{~b}$ shows the principle of operation of conductive elastomer strain gauges, where variation in conductive particle spacing is transduced as a change in resistance. In general, tensile strain causes increased resistance while compressive strain decreases mean particle spacing and decreases resistance. Figure $3 \mathrm{c}$ shows sample data collected with an Agilent 34401A multimeter from an elastomer strain gauge undergoing large $(\sim 25 \%)$ strain while being manually stretched.

Currently, a rigid substrate is required in order to bring heaters or sensors into close proximity to microfluidic channels and reaction chambers. Compliant total analysis systems such as required for implantation or use with wearable labs cannot be easily implemented in this way. In order to overcome these limitations, we have embedded conductive elastomer sensors along with microfluidic channels to allow detection of liquids, flow, organic solvents, as well as localized heating (Figure $1 \mathrm{~b}$ and Figure 4). To demonstrate this potential, we arranged an embedded sensor array along a channel (Figure 4a). The conductive portion serves as both a heater and sensor. The operating principle is basically that of a heated film flow meter as schematically represented in Figure $4 \mathrm{~b}$. When the sensor is heated above ambient and the resistance monitored, a change in output signifies a change in heat lost to the environment. With an excitation voltage of $6 \mathrm{~V}$, and only $1 \mu \mathrm{W}$ input power, a large $(\sim 10 \%)$ change in output voltage is measured when water is introduced into the channel (Figure $4 \mathrm{c}$ ). This change is due to heat loss to the fluid. Measurements are made using an Agilent 34401A multimeter. This can be used to detect fluid fronts or analyte plugs as commonly used in micro total analysis systems.

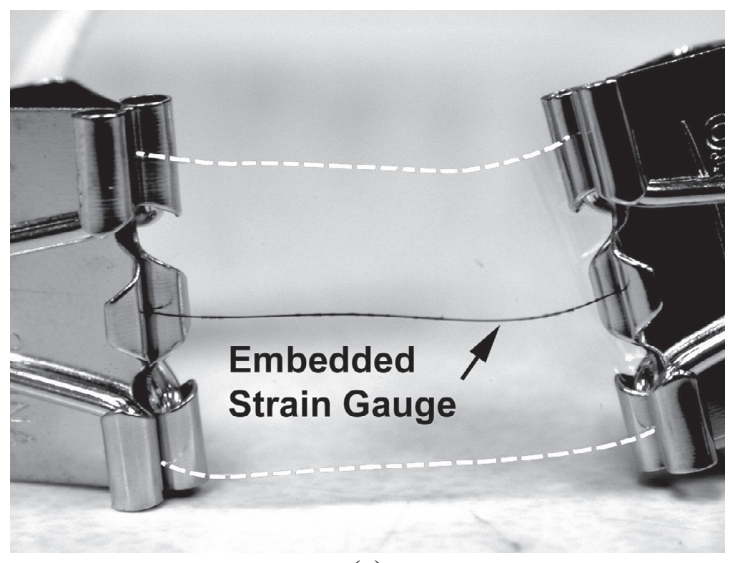

(a)

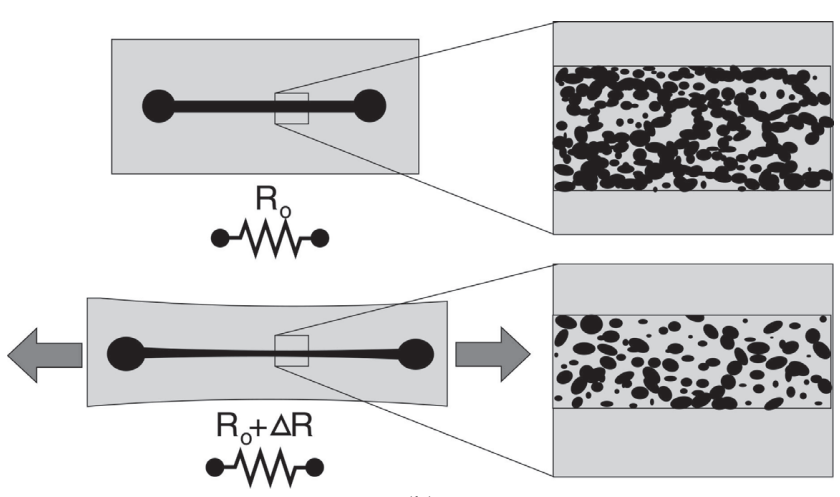

(b)

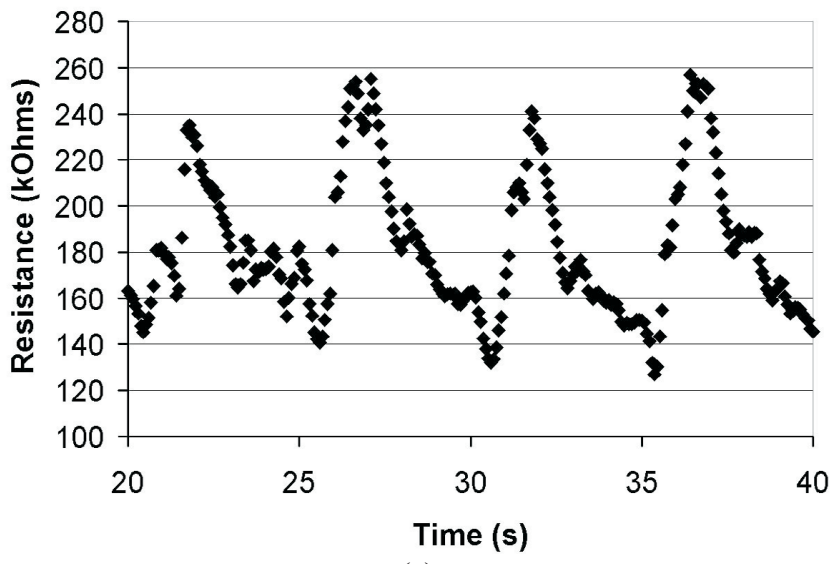

(c)

Figure 3: a) Photo of PDMS sheet with embedded strain gauge. Dashed white lines indicate perimeter of clear PDMS strip. b) Schematic showing operation of elastomer strain gauge operation. c) Sample data of MWNT strain gauge undergoing large ( 25\%) strain. 


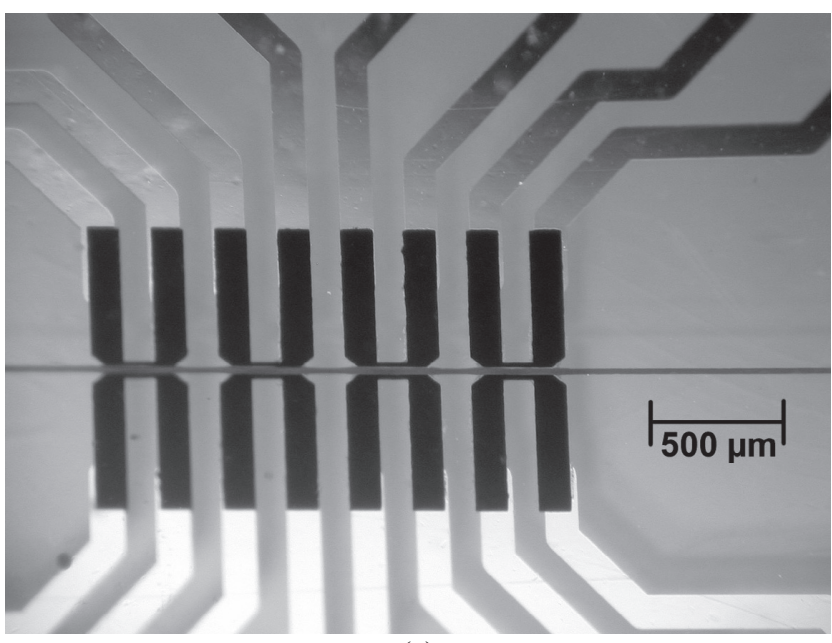

(a)

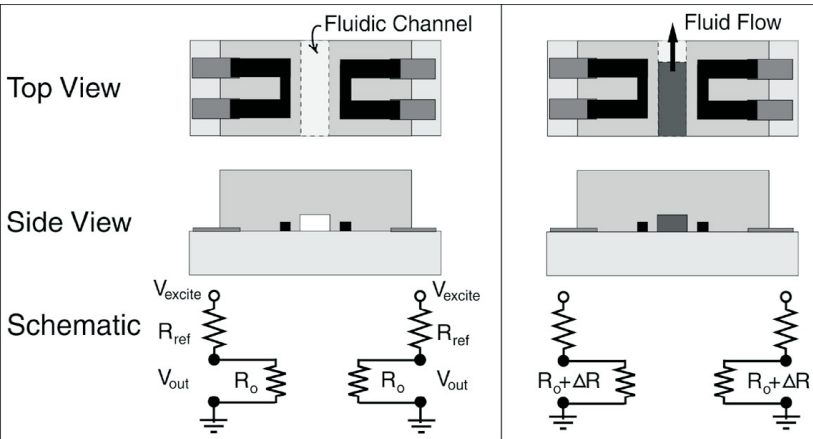

(b)

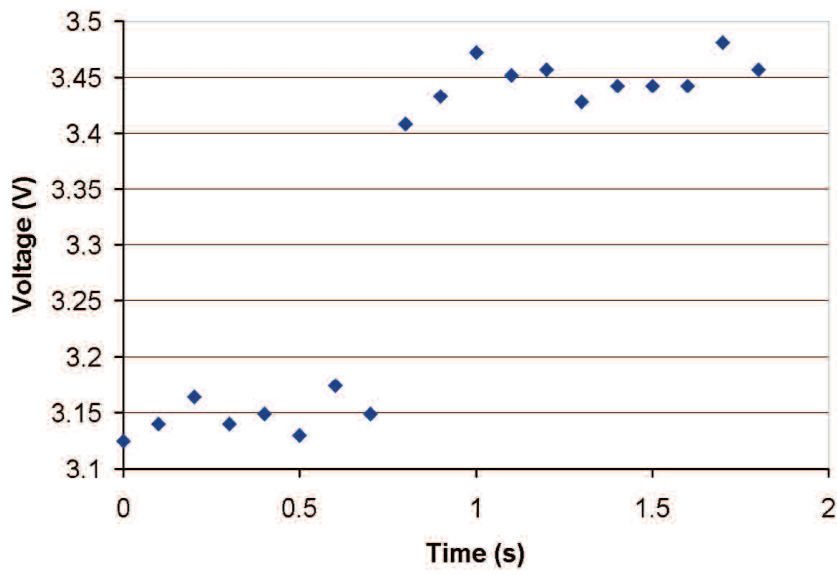

(c)

Figure 4: a) Micrograph of embedded flow sensors next to channel filled with red dye. b) Schematic showing operation of embedded flow sensors. An external reference resistor $\left(R_{\text {ref }}\right)$ is connected in series with the PDMS sensor $\left(R_{o}\right)$ and a current heats the sensor above ambient. When fluid flows in the channel heat is lost to the fluid, changing the sensor resistance and the output voltage. c) Chart showing change in output voltage (6V excitation) when fluid flows into channel.

By using multiple layers, more complex devices are also realized. For example, by combining two layers of elastomer with embedded electrodes (Figure 1) and orienting them orthogonal to each other a matrix of capacitive pressure sensors is created as shown in Figure 6a. The capacitance of a flat plate capacitor is

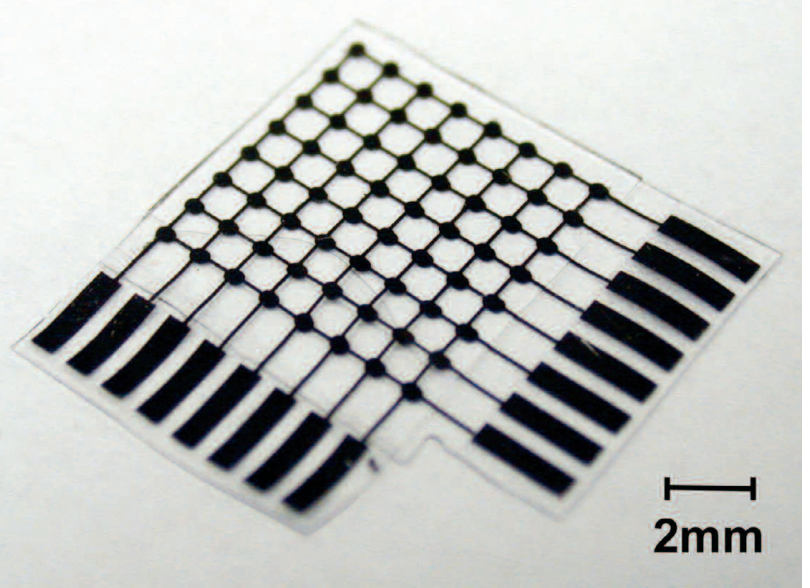

(a)

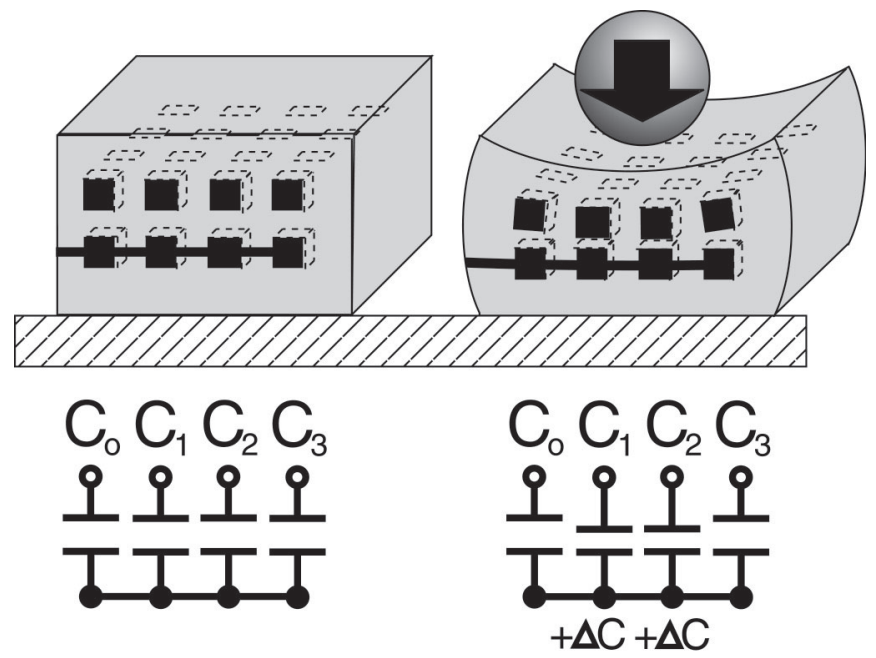

(b)

Figure 5: a) Photo of orthogonal embedded PDMS electrode layers, which results in an array of capacitive pressure sensors. b) Schematic showing operating principle of capacitive array.

proportional to electrode area and inversely proportional to electrode gap. Thus large area and small gap are desired, but using soft materials like PDMS presents a significant challenge for maintaining a small electrode gap. Previous efforts to make collapsible capacitive PDMS devices require larger gaps, numerous bonding steps, and subsequent large area. The presented device uses a PDMS filled capacitive gap of $4 \mu \mathrm{m}$ which gives it high stiffness compared to air-gap capacitive devices. However, the filled gap gives increased robustness to stiction, particles, and mechanical overload as well as increasing the baseline capacitance of the sensors. Air gap capacitors are possible using similar techniques to those used to create microfluidic channels (Figure 4).

Testing reveals that interrogating the row and column capacitance of the array allows imaging of contact with other objects. For example, when loaded by a $3 \mathrm{~mm}$ spherical indenter under a $500 \mathrm{~g}$ load and the capacitance measured with an Agilent 4263B LCR meter, the array changes capacitance as shown in Figure 6. Additionally, the negative-valued artifacts observed in Figure 6 can be eliminated by using electronics designed to interrogate multiplexed capacitive arrays. These circuits switch non-interrogated rows and columns to ground to minimize parasitic parallel capacitances. 


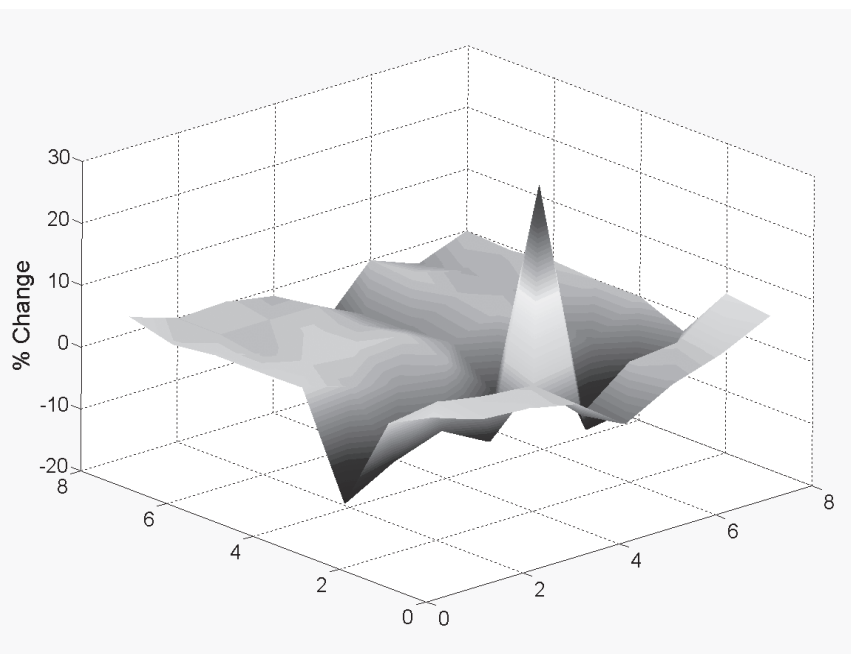

Figure 6: Graph of change in capacitance of array when loaded by a $3 \mathrm{~mm}$ spherical indenter. $X$ and $Y$ axes are the row and columns of the capacitive array.

\section{CONCLUSIONS}

We have demonstrated a new fabrication technique and approach to realizing all-elastomer MEMS devices. This is accomplished by combining micro patterning of conductive elastomer features with traditional spin casting and molding of insulating elastomers. Conductive elastomers are made functional by mixing with multi-walled carbon nanotubes. In this way we have created several new devices, including all-elastomer strain gauges, microfluidic systems with embedded elastomer sensors and heaters, and robust stretchable capacitive elastomer tactile sensors. The presented technique has further promise for soft biomedical applications, such as interocular pressure measurements, large strain measurements for smart-prosthetics and robotics, and compliant pathogen detection systems for wearable deployment.

\section{ACKNOWLEDGMENTS}

This work was funded by the Air Force Office of Scientific Research (AFOSR) Bio-Inspired Concept program and the Defense Advanced Research Projects Agency (DARPA) BioSenSE program.

\section{REFERENCES}

[1] J. Chen and N. Tsubokawa, "Novel Gas Sensor from Polymer-Grafted Carbon Black: Vapor Response of Electric Resistance of Conducting Composites Prepared from Poly(ethylene-block-ethylene-oxide)-Grafted Carbon Black," Journal of Applied Polymer Science, vol. 77, pp. 2437-2447, 1999.

[2] E. Segal, R. Tchoudakov, M. Narkis, and A. Siegmann, "Sensing of liquids by electrically conductive immiscible polypropylene / thermoplastic polyurethane blends containing carbon black," Journal of Polymer Science: B, vol. 41, pp. 142840, 2003.

[3] J. M. Engel, J. Chen, D. Bullen, and C. Liu, "Polyurethane Rubber as a MEMS Material: Characterization and Demonstration of an All-Polymer Two-Axis Artificial Haircell Flow Sensor," presented at IEEE International Conference on MEMS, 2005.

[4] T. Buma, M. Spisar, and M. O'Donnell, "A high frequency ultrasound array element using thermoelastic expansion in PDMS," presented at IEEE Ultrasonics Symposium, 2001.

[5] H. K. Lee, S. I. Chang, K. H. Kim, S. J. Kim, K. S. Yun, and E. Yoon, "A Modular Expandable Tactile Sensor Using Flexible Polymer," presented at IEEE International Conference on MEMS, 2005.

[6] Y. J. Jung, S. Kar, S. Talapatra, C. Soldano, G. Viswanathan, X. Li, Z. Yao, F. S. Ou, A. Avadhanula, R. Vajtai, S. Curran, O. Nalamasu, and P. M. Ajayan, "Aligned Carbon Nanotube-Polymer Hybrid Architectures for Diverse Flexible Electronic Applications," Nano Letters, vol. 6, pp. 413-418, 2006.

[7] K. Ryu, X. Wang, K. Shaikh, and C. Liu, "A method for precision patterning of silicone elastomer and its applications," Journal of Microelectromechanical Systems, vol. 13, pp. 568-575, 2004.

[8] J. M. Engel, J. Chen, N. Chen, S. Pandya, and C. Liu, "Multi-Walled Carbon Nanotube Filled Conductive Elastomers: Materials and Application to Micro Transducers," presented at IEEE International Conference on MEMS, 2006. 